\title{
EFEITO DA ADIÇÃO DE ÓLEOS AO ACARICIDA CYHEXATIN SOBRE O ÁCARO Brevipalpus phoenicis E NA RETENÇÃO DE CALDA POR FOLHAS DE CITROS ${ }^{1}$
}

\author{
DANIEL JÚNIOR DE ANDRADE², MARCELO DA COSTA FERREIRA³ \\ NATALI CALAZANÇA DOS SANTOS ${ }^{4}$
}

RESUMO-A adição de óleos à calda de pulverização, muitas vezes, é utilizada a campo sem o adequado conhecimento sobre a absorção do produto fitossanitário pelo alvo, retenção de calda e até mesmo sobre a praga e a cultura. $\mathrm{O}$ objetivo do trabalho foi avaliar o efeito da adição de óleos ao acaricida cyhexatin sobre o ácaro Brevipalpus phoenicis e na retenção de calda por folhas de citros. Avaliou-se a mortalidade de ácaros, utilizando-se de frutos de laranja com uma arena circundada com cola entomológica para confinar os ácaros. Adotou-se o delineamento inteiramente casualizado, em esquema fatorial, constituído pelos fatores: duas formulações de cyhexatin (WG e SC), dois tipos de óleo (mineral e vegetal) e duas concentrações dos óleos (0,5 e 1,0\%), e mais dois tratamentos adicionais (acaricidas não adicionados de óleo) e uma testemunha sem aplicação. A aplicação dos produtos foi realizada sobre frutos de laranja até além do ponto de escorrimento. Logo após a aplicação, transferiram-se 10 ácaros $B$. phoenicis para cada fruto. A contagem dos ácaros vivos, mortos e retidos na barreira adesiva foi realizada um dia após a aplicação. Para a determinação da quantidade de calda retida, utilizaram-se folhas de laranjeira, que foram pulverizadas até além do ponto de escorrimento, adotando-se os mesmos tratamentos e o delineamento estatístico mencionados para a avaliação da mortalidade de ácaros, com exceção da testemunha sem aplicação. Determinou-se a massa de líquido retido após a aplicação dos produtos por folha, com auxílio de balança de precisão. Verificou-se que um dia após a aplicação dos produtos, todos os tratamentos apresentaram mortalidade de $B$. phoenicis acima de 99\%. Dessa forma, a adição de óleo, seja mineral, seja vegetal, ao acaricida cyhexatin não afetou a eficácia biológica deste acaricida nas formulações SC e WG. A maior fuga de B. phoenicis para a barreira de cola foi verificada nos tratamentos com adição de óleos, em comparação ao cyhexatin aplicado isoladamente. A adição de óleo, seja mineral, seja vegetal, ao cyhexatin na calda de pulverização reduziu a quantidade máxima de líquido retido pelas folhas de citros, podendo contribuir para a redução da quantidade de calda necessária para uma boa cobertura da planta.

Termos para indexação: Leprose dos citros, ácaro da leprose, tecnologia de aplicação e adjuvante.

\section{EFFECT OF THE ADDITION OF OIL TO CYHEXATIN MITECIDE ON MITE Brevipalpus phoenicis AND IN SPRAY LIQUID RETENTION ON CITRUS LEAVES}

\begin{abstract}
The addition of adjuvant oils to spray is frequently used without any adequate knowledge about the product adsorption by the target, spray retention and the crop-pest interactions. This work aimed to evaluate the effect of adjuvant oils addition in the cyhexatin acaricide solution to Brevipalpus phoenicis control and the spray retention by citrus leaves. The mites mortality was evaluated in a certain area surrounded by entomological glue over orange fruits. It was performed a totally randomized design, in a factorial scheme. It was used two cyhexatin formulations: WG and CS; two adjuvant oils: vegetal and mineral over two concentrations $(0.5$ and $1.0 \%)$ plus two additional check treatments (acaricide without oil) and control (without spraying). Application was performed in orange fruits over the point of runoff. After application, ten B. phoenicis were transferred to each fruit. The count of alive, dead and glue barrier stucked mites was made one day after. In order to estimate the spray retention, a spray application over orange leaves was performed, sprayed up to the runoff point, using the same treatments and statistical design analysis that had been previously mentioned, except for control plot. The liquid mass over the leaves after spraying was weighed. It was verified, for all treatments, that the mite mortality reaches above $90 \%$, right away the day after application. Thus, both mineral and vegetal oil addition to cyhexatin acaricide spray did not affect its biological efficacy under CS and WG formulations. It was observed a larger number of mites stucked to the glue barrier in the treatments with oil addition compared to the treatments without it. The vegetal or mineral oil addiction to cyhexatin spray have reduced the maximum amount of liquid retention by the citrus leaves, which can contribute to reduce the essential spray amount in a great plant cover.
\end{abstract}

Index terms: Citrus leprosis, citrus leprosis mite, spray technology and adjuvant.

${ }_{1}^{1}$ (Trabalho 265-09). Recebido em: 25-11-2009. Aceito para publicação em: 22-09-2010.

${ }^{2}$ Doutorando em Agronomia (Entomologia Agrícola), Universidade Estadual Paulista, Faculdade de Ciências Agrárias e Veterinárias (FCAV/UNESP). Via de acesso Prof. Paulo Donato Castellane, s/n - CEP: 14884-970, Jaboticabal - SP. Bolsista FAPESP. E-mail: danieldwv@yahoo.com.br. Fone: (16) 32092640

${ }^{3}$ Prof. Adjunto, Departamento de Fitossanidade, FCAV/UNESP. E-mail: mdacosta@fcav.unesp.br

${ }^{4}$ Aluna de Graduação do curso de Agronomia, FCAV/UNESP. E-mail: natali.00@hotmail.com 


\section{INTRODUÇÃO}

A utilização de óleos minerais, vegetais ou derivados de petróleo na calda, para aplicação de produtos fitossanitários, visa a aumentar a eficiência de controle de pragas e doenças, quer protegendo a calda aplicada de intempéries, quer melhorando a interação entre a calda e o alvo.

No Brasil, diferentes marcas de óleos de origem mineral e vegetal estão disponíveis e apresentam potencial para uso em citros. Devido às suas características físico-químicas, os óleos podem ser misturados com produtos fitossanitários e nutrientes (RODRIGUES; CHILDERS, 2002).

Vargas e Roman (2006) ressaltam que, quando adicionados à calda de pulverização, os óleos podem aumentar a eficiência do produto fitossanitário, reduzir a deriva, retardar a evaporação da gota e atuar como espalhante e adesivo. De acordo com Childers (1997), acaricidas como abamectin e ethion são recomendados em mistura com óleos para a melhoria do efeito residual.

Todavia, acaricidas, como óxido de fenbutatin e pyridaben, têm redução comprovada de sua eficiência no controle do ácaro da leprose Brevipalpus phoenicis, quando associados ao óleo mineral ou ao vegetal na calda de pulverização (CHILDERS; SELHIME, 1983; CHILDERS, 1997; OLIVEIRA et al., 2003). De acordo com Furness (1981), a eficiência dos óleos adicionados aos acaricidas, no controle de pragas, está diretamente ligada a muitas variáveis, principalmente à concentração, ao momento da aplicação e à cobertura do alvo pela pulverização.

A adição de óleo mineral ou vegetal à calda de pulverização, em geral, altera a retenção em aplicações a alto volume, pois o excesso da calda que a folha não comporta, escorre, aumentando os custos de controle pela adição de mais um componente ao sistema, além de poder contribuir para contaminação do solo (MATUO, 1987; CUNHA et al., 2005). A partir desse fato, surge a hipótese de que menor retenção poderia acarretar redução do poder residual do acaricida, afetando sua eficiência (OLIVEIRA et al., 1997).

Diante disto, é fundamental adequar o volume da calda aplicada para satisfazer à necessidade de molhamento foliar, com o melhor aproveitamento de recursos. Assim, o sucesso do tratamento fitossanitário depende da utilização de produtos com eficácia comprovada, aliada à tecnologia específica para a aplicação (RODRIGUES et al., 2003). Além disso, para se obter controle eficiente de pragas, é necessário um conhecimento multidisciplinar para se compreender a complexidade dos fatores intrínsecos envolvidos (PEREIRA, 1987).

O objetivo deste trabalho foi avaliar o efeito da adição de óleos mineral e vegetal ao acaricida cyhexatin sobre a mortalidade de ácaros $B$. phoenicis e a retenção de líquido por folhas de citros.

\section{MATERIAL E MÉTODOS}

Os experimentos foram realizados no Laboratório de Acarologia do Departamento de Fitossanidade/UNESP, Câmpus de Jaboticabal-SP, nos meses de janeiro a abril de 2009.

Para dar início à criação-estoque de ácaros da leprose, foram coletados frutos de laranja da variedade Pera, com sintomas de leprose e infestados com B. phoenicis, em pomar de citros da região de Jaboticabal, que há 12 meses não recebiam pulverização com produtos fitossanitários. Escolheram-se frutos que também apresentavam sintomas de leprose e apresentassem sintomas de verrugose, pois o ácaro tem preferência por frutos com superfície irregular (ALBUQUERQUE et al., 1997). No laboratório, os frutos foram lavados com água corrente, secados à sombra e parcialmente parafinados, exceto por uma área de aproximadamente $10 \mathrm{~cm}^{2}$, que foi circundada com cola entomológica (Cola Tatoo $\left.{ }^{\circledR}\right)$ para conter os ácaros.

Os frutos foram dispostos em bandejas plásticas e mantidos em câmara climatizada a $25 \pm 1^{\circ} \mathrm{C}$, UR de $60 \pm 5 \%$ e fotofase de 14 horas. Quando necessário, os frutos em início de deterioração foram substituídos, sendo a transferência dos ácaros realizada por meio do contato entre o fruto a ser substituído e o novo fruto.

Realizaram-se dois experimentos, sendo um para a avaliação da mortalidade de ácaros $B$. phoenicis e outro para avaliar a retenção de calda fitossanitária por folhas de citros. Para a avaliação da mortalidade, foram colhidos frutos de laranja da variedade Pera, com sintomas de verrugose, em um pomar pertencente à UNESP, Câmpus de Jaboticabal, com 12 meses sem pulverizações com produtos fitossanitários.

O delineamento adotado no experimento foi o inteiramente casualizado, em esquema fatorial, constituído pelos fatores formulações de cyhexatin (Sipcatin 500 SC e Acarstin WG), óleo mineral ou vegetal e concentração dos óleos a 0,5 e 1,0\% (2 x 2 x $2)+2$ tratamentos adicionais (acaricidas sem adição de óleo) e uma testemunha sem aplicação, totalizando 11 tratamentos, repetidos 7 vezes, sendo cada repetição composta por um fruto de laranja. Os tratamentos 
estabelecidos, expressos em $\mathrm{mL}$ ou g p.c./100 L de água destilada, encontram-se na Tabela 1 .

A pulverização com as caldas sobre os frutos de laranja foi realizada com dois bicos de cone vazio, modelo TXVK2, posicionados lateralmente, apoiados em um suporte e dispostos dentro de uma bandeja de metal para recolher o excesso de calda. Pulverizou-se um fruto por vez, até além do ponto de escorrimento, utilizando-se de um suporte para manter o fruto entre os dois bicos durante a aplicação dos produtos. Após a aplicação, os frutos receberam o mesmo processo descrito no preparo dos frutos da criação-estoque. Porém, foi deixada nos frutos uma área menor $\left(5 \mathrm{~cm}^{2}\right)$ sem parafina, que foi circunda com cola entomológica (Cola Tatoo $\left.{ }^{\circledR}\right)$.

Com auxílio de pincel de apenas um pelo e microscópio estereoscópico, transferiram-se para cada fruto, após a aplicação dos produtos, 10 ácaros adultos de $B$. phoenicis, procedentes da criaçãoestoque. Esses frutos foram mantidos em câmara climatizada, nas mesmas condições de temperatura, umidade relativa e fotofase citadas. Foram realizadas avaliações de mortalidade um dia após a transferência dos ácaros, quantificando-se o número de ácaros mortos, vivos e retidos na barreira adesiva de cada parcela experimental. A realização de outras avaliações não foi necessária devido à mortalidade dos ácaros ter atingido praticamente 100\% (acima de 99\%) em todos os tratamentos.

Para avaliar a retenção de calda, adotou-se a metodologia descrita por Matuo et al. (1989), nos quais foram coletadas folhas de laranja com tamanhos visualmente semelhantes em um pomar da variedade Pera, com quatro anos de idade, pertencente ao Câmpus da UNESP de Jaboticabal.

A aplicação da calda de pulverização sobre as folhas foi realizada seguindo a mesma metodologia citada anteriormente para a aplicação dos produtos sobre os frutos. Dessa forma, cada folha foi posicionada verticalmente em um suporte colocado sobre o prato de uma balança digital (Modelo Mettler PC 440, com precisão de $1 \mathrm{mg}$ ), procedendo-se a tara da balança. Na sequência, procedeu-se à aplicação da calda de pulverização sobre a folha, de modo a permitir que a calda atingisse uniformemente as superfícies abaxial e adaxial da folha. Foram utilizados dois bicos de cone cheio, modelo TXVK2. Imediatamente após cessar o escorrimento do excesso de calda, foi anotada a massa da calda retida em miligramas (mg). Anotouse, portanto, o peso da folha antes e após a aplicação, sendo a massa de calda obtida pela diferença entre os pesos.

Adotaram-se os mesmos tratamentos e delineamento estatístico mencionados para a avaliação da mortalidade de ácaros, com exceção da testemunha sem aplicação de produto fitossanitário, sendo cada repetição constituída por uma folha de laranja (Tabela 1).

Para a determinação da área de cada folha, utilizou-se a técnica da digitalização da imagem por meio da captura da imagem em scanner (SILVA et al., 2008), e, com auxílio do software QUANT v.01 (VALE et al., 2001), foi calculada a área foliar e, assim, a retenção máxima de líquido foi expressa em $\mathrm{mL}$ de calda por $\mathrm{m}^{2}$.

Os dados obtidos em ambos os experimentos foram transformados em $\ln (\mathrm{x}+5)$ e submetidos à análise de variância, pelo teste $\mathrm{F}$, e as médias, comparadas pelo teste de Tukey, a 5\% de probabilidade. A porcentagem de mortalidade de ácaros dos tratamentos foi calculada pela fórmula de Abbott (1925).

\section{RESULTADOS E DISCUSSÃO}

\section{Avaliação da mortalidade de B. phoenicis}

- Na avaliação realizada 1 dia após a transferência dos ácaros para os frutos, todos os tratamentos diferiram estatisticamente da testemunha, mas não entre si, e apresentaram percentuais de mortalidade, excluindo-se os ácaros retidos na cola, acima de $99 \%$, razão pela qual não se justificou a realização de mais avaliações (Tabela 2). Diversos trabalhos realizados, principalmente no Brasil (OLIVEIRA et al., 1997; OLIVEIRA et al., 2003; FERREIRA, 2003; FERNANDES et al., 2008), comprovaram a eficiência do cyhexatin acima de $90 \%$, um dia após a aplicação, no controle do ácaro B. phoenicis.

A princípio, esses resultados não justificariam a adição dos óleos ao cyhexatin, entretanto estes podem ser utilizados como subsídios para as próximas pesquisas, pois admite-se que a adição de óleo mineral ou vegetal à calda de pulverização não afetou negativamente o efeito biológico do cyhexatin.

Com relação aos ácaros retidos na barreira adesiva de cola, foram verificadas diferenças entre os tratamentos. Pode-se observar, na Tabela 3, que o fator tipo de óleo apresentou significância estatística com relação aos ácaros retidos na cola, sendo que o óleo vegetal, em média, apresentou o maior número de ácaros na cola em relação ao óleo mineral.

No que se refere às interações, verificou-se que a interação entre o fator acaricida e o fator tipo de óleo foi significativa. Observou-se que o acaricida cyhexatin WG, adicionado de óleo mineral, apresentou a maior média de ácaros retidos na cola, em relação ao cyhexatin SC adicionado de óleo 
mineral, diferindo estatisticamente. Verificou-se, também, que a adição de óleo vegetal ao cyhexatin $\mathrm{SC}$ apresentou diferença estatística em relação à adição de óleo mineral (Tabela 4).

Ressalta-se que o número de ácaros retidos na barreira adesiva, nos tratamentos adicionados de óleos, foi de modo geral superior à testemunha e aos tratamentos em que se empregaram somente os acaricidas não adicionados de óleo. Constatou-se que todos os tratamentos com o emprego de óleo mineral ou vegetal, com exceção do cyhexatin SC + óleo mineral $(0,5 \% \mathrm{v} / \mathrm{v})$, apresentaram percentuais de ácaros retidos na cola superior a $30 \%$ em relação ao total de ácaros (Tabela 2).

Desta forma, pode-se admitir que essa maior fuga de ácaros para a barreira adesiva seja devida a ácaros que não se contaminaram com o ingrediente ativo, ou se contaminaram com quantidade insuficiente para ocasionar sua morte, acarretando, no entanto, repelência e/ou irritabilidade aos ácaros, o que resultou na maior fuga deles para a barreira adesiva. Oliveira et al. (2003) verificaram, por meio do teste de índice de iodo (método de Hübl), que moléculas de diversos acaricidas misturados com óleo vegeral, entre os quais o cyhexatin, foram incorporadas por ligações insaturadas do óleo. Todavia, os autores não atribuíram esse fato à redução da eficiência dos acaricidas, após a mistura com óleo, no controle do ácaro $B$. phoenicis.

\section{Retenção de calda por folhas de citros} - Analisando os resultados da Tabela 4, pode-- se verificar que houve diferenças significativas para os fatores avaliados em relação à capacidade máxima de retenção de calda. Constatou-se que houve diferença entre os acaricidas, sendo retida maior quantidade de calda nas folhas tratadas com cyhexatin WG do que naquelas tratadas com cyhexatin SC

Sendo um dos critérios para estimar a eficiência de uma aplicação de produto fitossanitário, a retenção de produto pela superfície do alvo pode variar principalmente devido às características da espécie vegetal e do estádio de desenvolvimento da planta (FERREIRA, 2003). Dessa forma, o que não é retido pelo alvo é perdido principalmente por escorrimento, reduzindo a eficiência da aplicação devido ao aumento dos custos e da contaminação do ambiente. Como foi verificado, entretanto, a formulação dos produtos aplicados também interagiu com a superfície vegetal, afetando a retenção da calda.

Neste contexto, Matuo et al. (1989) verificaram que a adição de adjuvantes à calda de pulverização proporcionou menor retenção do líquido nas folhas de laranjeira, em pulverizações a alto volume. Todavia, a adição de adjuvantes à calda de pulverização pode aumentar a superfície coberta pela calda, devido à "quebra" da tensão superficial da água, o que permite maior espalhamento das gotas e, com isso, pode possibilitar reduções do volume de calda.

Observou-se que o óleo mineral proporcionou menor retenção de calda em relação ao óleo vegetal (Figura 1). Essa menor retenção deveu-se à maior redução da tensão superficial da calda, incrementando o escorrimento e, consequentemente, podendo reduzir a quantidade de ingrediente ativo sobre a superfície da folha. Oliveira et al. (2003) acreditam que essas diferenças observadas entre o óleo mineral e o vegetal sejam, provavelmente, devidas à composição dos mesmos. Enquanto o óleo mineral é proveniente da destilação do petróleo e representa uma fração mais homogênea, o óleo vegetal é composto por diferentes gorduras, quimicamente distintas, normalmente insaturadas e heterogêneas.

No que tange às concentrações dos óleos, verificou-se que a capacidade de retenção de calda foi maior quando se utilizou a menor concentração de óleo mineral e/ou vegetal $(0,5 \%)$. Resultados semelhantes foram obtidos por Ocampo-Ruiz e Matuo (1994), que estudaram a retenção do acaricida propargite em folhas de citros, em mistura com três concentrações de adjuvantes, e verificaram que a quantidade retida pelas folhas foi inversamente proporcional ao aumento da concentração do adjuvante à calda.

$\mathrm{Na}$ interação dos fatores acaricidas e tipo de óleo, observou-se que a retenção de calda proporcionada pela adição de óleo vegetal ao acaricida cyhexatin $\mathrm{SC}$ foi superior à adição de óleo mineral e foram diferentes estatisticamente. A retenção de calda no tratamento com óleo vegetal em mistura com o cyhexatin SC foi aproximadamente de $90 \mathrm{~mL} / \mathrm{m}^{2}$, enquanto o óleo mineral em mistura com cyhexatin $\mathrm{SC}$ foi de $60 \mathrm{~mL} / \mathrm{m}^{2}$.

Entretanto, os óleos, em mistura com produtos fitossanitários ou aplicados isoladamente, devem ser empregados de maneira criteriosa, em razão de que o uso de forma inadequada, como, por exemplo, o emprego de produtos fitossanitários incompatíveis com determinado tipo de óleo, ou até mesmo a utilização de concentrações inadequadas do óleo na calda de pulverização, pode resultar em redução da eficiência de controle. Outro aspecto importante refere-se às reações de sensibilidade das plantas (fitotoxicidade), decorrente de aplicações de óleos. Assim, antes de optar pelo uso de óleos adicionados à calda de pulverização, devem-se sempre buscar informações sobre a suscetibilidade da cultura, sobre as dosagens compatíveis com o estádio fenológico da planta, a compatibilidade com 
os produtos fitossanitários, o ajuste entre dosagem e época do ano, o estado nutricional e fisiológico da cultura, a característica e a qualidade do óleo (RODRIGUES; CHILDERS, 2002).

Como o efeito da adição de óleos ainda não é conhecido para muitos produtos, a condução de estudos que visem a elucidar principalmente as possíveis reações fitotóxicas e as reduções da eficiência dos produtos, é extremamente importante e necessária, para uma adequada recomendação dos óleos em programas de manejo de pragas na cultura dos citros.

TABELA 1 - Relação dos tratamentos utilizados no experimento de avaliação da mortalidade de ácaros Brevipalpus phoenicis.

\begin{tabular}{|c|c|c|}
\hline \multirow{2}{*}{ Tratamentos } & \multirow{2}{*}{ Produtos } & Dosagens \\
\hline & & mL ou g p.c./100L + (v/v) \\
\hline 1 & cyhexatin $^{1}+$ óleo mineral ${ }^{3}$ & $50+0,5 \%$ \\
\hline 2 & cyhexatin $^{1}+$ óleo mineral & $50+1,0 \%$ \\
\hline 3 & cyhexatin $^{1}+$ óleo vegetal $^{4}$ & $50+0,5 \%$ \\
\hline 4 & cyhexatin $^{1}+$ óleo vegetal & $50+1,0 \%$ \\
\hline 5 & cyhexatin $^{2}+$ óleo mineral & $40+0,5 \%$ \\
\hline 6 & cyhexatin $^{2}+$ óleo mineral & $40+1,0 \%$ \\
\hline 7 & cyhexatin $^{2}+$ óleo vegetal & $40+0,5 \%$ \\
\hline 8 & cyhexatin $^{2}+$ óleo vegetal & $40+1,0 \%$ \\
\hline 9 & cyhexatin $^{1}$ & 50 \\
\hline 10 & cyhexatin $^{2}$ & 40 \\
\hline 11 & Testemunha (aplicação de água destilada) & - \\
\hline
\end{tabular}

TABELA 2 - Número médio de ácaros Brevipalpus phoenicis vivos, mortos e retidos na barreira de cola entomológica na avaliação realizada um dia após a aplicação dos produtos.

\begin{tabular}{|c|c|c|c|c|c|}
\hline \multirow{2}{*}{ Tratamentos } & \multirow{2}{*}{ Produtos } & \multicolumn{3}{|c|}{ Número de ácaros - $1 \mathbf{D A A}^{5}$} & \multirow{2}{*}{$\begin{array}{c}\text { Eficiência } \\
(\%)\end{array}$} \\
\hline & & mortos & vivos & retidos na cola & \\
\hline 1 & cyhexatin $^{1}+$ óleo mineral ${ }^{3}$ & 8,0 & $0,0 \mathrm{~b}$ & $0,9 \mathrm{c}$ & 100 \\
\hline 2 & cyhexatin $^{1}+$ óleo mineral & 7,1 & $0,0 \mathrm{~b}$ & $2,9 \mathrm{abc}$ & 100 \\
\hline 3 & cyhexatin $^{1}+$ óleo vegetal ${ }^{4}$ & 4,7 & $0,0 \mathrm{~b}$ & $5,3 \mathrm{a}$ & 100 \\
\hline 4 & cyhexatin $^{1}+$ óleo vegetal & 5,9 & $0,0 \mathrm{~b}$ & $4,0 \mathrm{ab}$ & 100 \\
\hline 5 & cyhexatin $^{2}+$ óleo mineral & 5,8 & $0,0 \mathrm{~b}$ & $4,1 \mathrm{ab}$ & 100 \\
\hline 6 & cyhexatin $^{2}+$ óleo mineral & 5,9 & $0,0 \mathrm{~b}$ & $4,1 \mathrm{ab}$ & 100 \\
\hline 7 & cyhexatin $^{2}+$ óleo vegetal & 6,7 & $0,0 \mathrm{~b}$ & $3,3 \mathrm{ab}$ & 100 \\
\hline 8 & cyhexatin ${ }^{2}+$ óleo vegetal & 6,3 & $0,4 \mathrm{~b}$ & $3,3 \mathrm{ab}$ & 99,4 \\
\hline 9 & cyhexatin $^{1}$ & 9,1 & $0,0 \mathrm{~b}$ & $0,9 \mathrm{c}$ & 100 \\
\hline 10 & cyhexatin $^{2}$ & 9,2 & $0,0 \mathrm{~b}$ & $0,8 \mathrm{c}$ & 100 \\
\hline 11 & $\begin{array}{l}\text { Testemunha (aplicação de água } \\
\text { destilada) }\end{array}$ & 1,3 & $7,8 \mathrm{a}$ & $0,9 \mathrm{c}$ & - \\
\hline
\end{tabular}

${ }^{1}$ Sipcatin ${ }^{\circledR} 500$ SC. ${ }^{2}$ Acarstin ${ }^{\circledR}$ WG. ${ }^{3}$ óleo mineral Assist ${ }^{\circledR} .{ }^{4}$ óleo vegetal Veget'oil $®$

DAA $^{5}$ - Dia após a aplicação.

*Médias seguidas por letras iguais na coluna, não diferem entre si, pelo teste de Tukey, a 5\% de probabilidade. 
TABELA 3 - Resultado do teste F para o número de ácaros retidos na barreira adesiva, no experimento de avaliação da mortalidade de Brevipalpus phoenicis. Fatorial $(2 \times 2 \times 2)+2$ tratamentos adicionais (acaricidas sem adição de óleo) e um tratamento sem aplicação.

\begin{tabular}{|c|c|c|}
\hline Variáreis & & Teste de Tukey \\
\hline \multirow{2}{*}{ Tipo de formulação } & Sipcatin SC & Acarstin WG \\
\hline & $0,89 \mathrm{a}$ & $0,92 \mathrm{a}$ \\
\hline \multirow{2}{*}{ Tipo de óleo } & Mineral & Vegetal \\
\hline & $0,88 \mathrm{~b}$ & $0,94 \mathrm{a}$ \\
\hline \multirow{2}{*}{ Concentração do óleo } & $0,5 \%$ & $1,0 \%$ \\
\hline & $0,90 \mathrm{a}$ & $0,91 \mathrm{a}$ \\
\hline Água destilada & \multicolumn{2}{|c|}{$0,85 \mathrm{a}$} \\
\hline Sipcatin SC (sem óleo) & \multicolumn{2}{|c|}{$0,77 \mathrm{a}$} \\
\hline Acarstin WG (sem óleo) & \multicolumn{2}{|c|}{$0,75 \mathrm{a}$} \\
\hline VARIÁVEIS & \multicolumn{2}{|c|}{ TESTE $\mathbf{F}$} \\
\hline Tipo de formulação (A) & \multicolumn{2}{|c|}{$1,61^{\mathrm{ns}}$} \\
\hline Tipo de óleo (B) & \multicolumn{2}{|c|}{$4,83^{*}$} \\
\hline Concentração do óleo (C) & \multicolumn{2}{|c|}{$0,47^{\mathrm{ns}}$} \\
\hline \multicolumn{3}{|l|}{ INTERAÇÕES } \\
\hline $\mathrm{A} \times \mathrm{B}$ & \multicolumn{2}{|c|}{$14,18^{* *}$} \\
\hline $\mathrm{AXC}$ & \multicolumn{2}{|c|}{$0,14^{\mathrm{ns}}$} \\
\hline $\mathrm{B} \times \mathrm{C}$ & \multicolumn{2}{|c|}{$3,74^{\mathrm{ns}}$} \\
\hline $\mathrm{A} \times \mathrm{B} \times \mathrm{C}$ & \multicolumn{2}{|c|}{$2,79^{\mathrm{ns}}$} \\
\hline$C V^{1}(\%)$ & \multicolumn{2}{|c|}{11,53} \\
\hline
\end{tabular}

Médias seguidas por letras iguais na mesma linha e na coluna, para os tratamentos com água destilada, cyhexatin SC e WG (sem óleo), não diferem entre si, pelo teste de Tukey, a $5 \%$ de probabilidade. Para a comparação das médias, os dados originais foram transformados em $\ln (\mathrm{x}+5)$. ${ }^{* *}$ Significativo pelo teste $\mathrm{F}$, a $5 \%$ de probabilidade. ns - Não significativo. ${ }^{1}$ Coeficiente de variação $(\%)$.

TABELA 4 - Média de ácaros Brevipalpus phoenicis retidos na barreira adesiva, nas interações dos fatores acaricidas e tipo de óleo.

\begin{tabular}{ccc}
\hline \multirow{2}{*}{ Acaricidas } & \multicolumn{2}{c}{${ }^{\mathbf{1}}$ Média de ácaros retidos na cola } \\
\cline { 2 - 3 } & \multicolumn{2}{c}{ Tipo de óleo } \\
\cline { 2 - 3 } & mineral & vegetal \\
\hline Cyhexatin SC & $1,87 \mathrm{~b} \mathrm{~B}$ & $4,62 \mathrm{a} \mathrm{A}$ \\
Cyhexatin WG & $4,12 \mathrm{a} \mathrm{A}$ & $3,25 \mathrm{a} \mathrm{A}$ \\
\hline
\end{tabular}

${ }^{1}$ Dados originais foram transformados em $\ln (x+5)$. Médias seguidas pela mesma letra, minúscula na coluna e maiúscula na linha, não diferem entre si, pelo teste de Tukey, a $5 \%$ de probabilidade. 
TABELA 5 - Resultado do teste F para a retenção de líquido pelas folhas de citros, com áreas foliares estimadas pelo método da digitalização da imagem. Fatorial 2 × 2 × 2 .

\begin{tabular}{|c|c|c|}
\hline Variáveis & \multicolumn{2}{|c|}{ Teste de Tukey } \\
\hline \multirow{2}{*}{ Tipo de formulação } & Sipcatin SC & Acarstin WG \\
\hline & $4,3 \mathrm{~b}$ & $4,5 \mathrm{a}$ \\
\hline \multirow{2}{*}{ Tipo de óleo } & Mineral & Vegetal \\
\hline & $4,3 \mathrm{~b}$ & $4,5 \mathrm{a}$ \\
\hline \multirow{2}{*}{ Concentração do óleo } & $0,5 \%$ & $1,0 \%$ \\
\hline & $4,4 \mathrm{a}$ & $4,3 \mathrm{~b}$ \\
\hline VARIÁVEIS & \multicolumn{2}{|c|}{ TESTE $\mathbf{F}$} \\
\hline Tipo de formulação (A) & \multicolumn{2}{|c|}{$23,20 * *$} \\
\hline Tipo de óleo (B) & \multicolumn{2}{|c|}{$14,94 * *$} \\
\hline Concentração do óleo (C) & \multicolumn{2}{|c|}{$7,21 * *$} \\
\hline \multicolumn{3}{|l|}{ INTERAÇÕES } \\
\hline $\mathrm{AXB}$ & \multicolumn{2}{|c|}{$29,96 * *$} \\
\hline $\mathrm{AXC}$ & \multicolumn{2}{|c|}{$2,15^{\mathrm{ns}}$} \\
\hline $\mathrm{B} \times \mathrm{C}$ & \multicolumn{2}{|c|}{$0,00^{\mathrm{ns}}$} \\
\hline $\mathrm{A} \times \mathrm{B} \times \mathrm{C}$ & \multicolumn{2}{|c|}{$4,50^{*}$} \\
\hline $\mathrm{CV}^{1}(\%)$ & \multicolumn{2}{|c|}{4,14} \\
\hline
\end{tabular}

Médias seguidas por letras iguais na mesma linha não diferem entre si, pelo teste de Tukey, a 5\% de probabilidade. Para a comparação das médias, os dados originais foram transformados em $\ln (\mathrm{X}+5)$. ${ }^{* *}$ Significativo pelo teste $\mathrm{F}$, a $5 \%$ de probabilidade. ${ }^{\text {ns }}$ Não significativo. ${ }^{1}$ Coeficiente de variação $(\%)$.

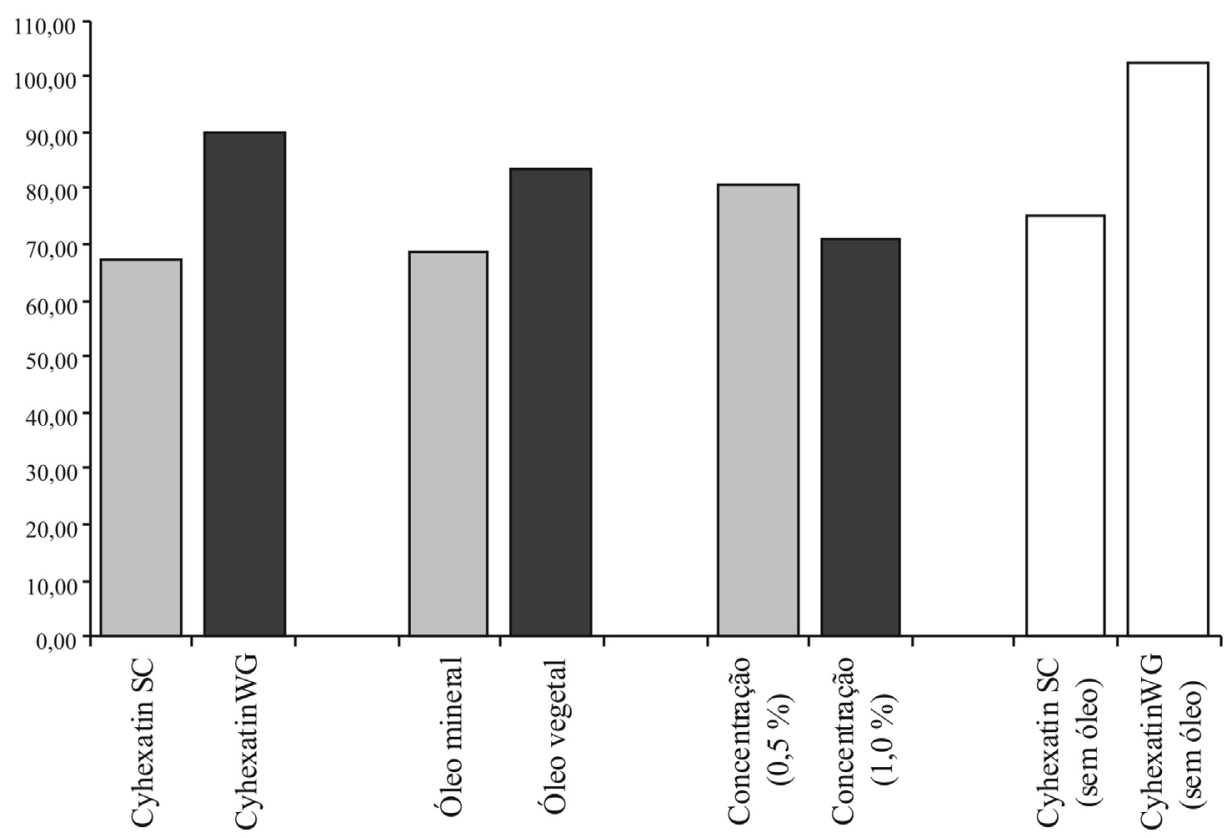

Variáveis

FIGURA 1 - Retenção máxima de calda por folhas de citros em função das formulações do cyhexatin (SC e WG), do tipo de óleo (mineral e vegetal), das concentrações dos óleos $(0,5$ e $1,0 \%)$ e dos acaricidas sem adição de óleo, no experimento de avaliação da retenção de calda. 


\section{CONCLUSÕES}

1- A adição de óleo mineral ou vegetal ao acaricida cyhexatin na calda de pulverização não afeta a eficácia biológica deste acaricida nas formulações $\mathrm{SC}$ e WG, sobre o ácaro Brevipalpus phoenicis.

2-Verifica-se a maior fuga de Brevipalpus phoenicis para a barreira de cola nos tratamentos com adição de óleo, em comparação ao cyhexatin aplicado isoladamente.

3-A adição de óleo ao cyhexatin, à calda de pulverização, seja mineral, seja vegetal, diminui a quantidade máxima de líquido retido pelas folhas, podendo contribuir para a redução da quantidade de calda necessária para uma boa cobertura da planta.

\section{REFERÊNCIAS}

ABBOTT, W.S. A method of computing the effectiveness of an insecticide. Journal of Economic Entomology, Lanham, v.18, p.265-267, 1925.

ALBUQUERQUE, F.A.; OLIVEIRA, C.A.L. de; BARRETO, M. Estudos da relação entre as incidências de verrugose da laranja-doce e leprose dos citros em frutos de laranja-pera. Científica, Jaboticabal, v.25, n.2, p.393-402. 1997.

CHILDERS, C.C. "Nexter" (pyridaben) - a new miticide for use on Florida citrus. Proceedings of the Florida State Horticultural Society, Tallahassee, v.110, p.59-64, 1997

CHILDERS, C.C.; SELHIME, A.G. Reduced efficacy of fenbutatin-oxide in combination with petrolicess oil controling the citrus rust mite Phyllocoptruta oleivora. Florida Entomologist, Gainesville, v.66, p.310-319, 1983.

CUNHA, J.A.R.; TEIXEIRA, M.M.; VIEIRA, R.F.; FERNANDES, H.C. Deposição e deriva de calda fungicida aplicada em feijoeiro, em função de bico de pulverização e de volume de calda. Revista Brasileira de Engenharia Agrícola e Ambiental, Campina Grande, v. 9, n. 1, p. 133-138, 2005.

FERNANDES, A.P.; OLIVEIRA, C.A.L. de; FERREIRA, M.C. Eficiência de acaricidas sobre populações de Brevipalpus phoenicis (Geijskes) (Acari Tenuipalpidae) de citros e cafeeiro. Revista de Agricultura, Piracicaba, v.83, n.1, p.45-55, 2008.
FERREIRA, M.C. Caracterização da cobertura de pulverização necessária para controle do ácaro Brevipalpus phoenicis (G., 1939) em citros. 2003. 64 f. Tese (Doutorado em Produção Vegetal) - Faculdade de Ciências Agrárias e Veterinárias, Universidade Estadual Paulista, Jaboticabal, 2003.

FURNESS, G.O. The role of petroleum oil sprays in the pest management programs on citrus in Australia. Proceedings of the International Society of Citriculture, Washington, v.2, p.607- 611, 1981.

MATUO, T. Enfoque multidisciplinar da tecnologia de aplicação de defensivos agrícolas. In: MATUO, T.; FERREIRA, M.E.; CARVALHO, R.P.L.; TAMAKI, $T$. Tecnologia de aplicação de defensivos agrícolas Jaboticabal: FUNEP, 1987. p.3-11.

MATUO, T.; NAKAMURA, S.H.; ALMEIDA, A. Efeito de alguns adjuvantes da pulverização nas propriedades físicas do líquido. Summa Phytopathology, Botucatu, v.15, p.163-173, 1989.

OCAMPO-RUIZ, R.A.; MATUO, T. Efeito de espalhantes-adesivos na retenção e na ação do propargite sobre Brevipalpus phoenicis (Geijs.) em folhas de citros. Anais da Sociedade Entomológica do Brasil, Campinas, v.23, n.2, p.265-270, 1994

OLIVEIRA C.A.L.de; MATUO, T.; SANTOS JUNIOR, J.E.; TOLEDO, M.C. Efeito de espalhanteadesivo na eficiência dos acaricidas propargite e cyhexatin no controle do Brevipalpus phoenicis (Geijskes) (Acari: Tenuipalpidae) em citros. Anais da Sociedade Entomológica do Brasil, Campinas v.26, n.3, p.487-493, 1997.

OLIVEIRA, C.P.; OLIVEIRA, C.A.L.de; MELO, W.J. Efeito da adição de óleos mineral e vegetal a acaricidas no controle do ácaro-da-leprose-dos-citros Brevipalpus phoenicis (Geijskes) (Acari: Tenuipalpide). Revista Brasileira de Fruticultura, Jaboticabal, v.25, n.2, p.224-226, 2003.

PEREIRA, J.L. Tecnologia de aplicação de defensivos - fatores intrínsecos. In: MATUO, T.; FERREIRA, M.E.; CARVALHO, R.P.L.; TAMAKI, T. Tecnologia de aplicação de defensivos agrícolas. Jaboticabal: FUNEP, 1987. p.13-40.

RODRIGUES, J.C.V.; CHILDERS, C.C. Óleos no manejo de pragas e doenças em citros. Laranja, Cordeirópolis, v.23, n.1, p.77-100, 2002.

RODRIGUES, J.C.V.; KITAJIMA, E.W.; CHIL- 
DERS, C.C.; CHAGAS, C.M. Citrus leprosis virus vectored by Brevipalpus phoenicis (Acari: Tenuipalpidae) on citrus in Brasil. Experimental and Applied Acarology, Amsterdam, v.30, n.1, p.161179, 2003.

SILVA, A.R.; LEITE, M.T.; FERREIRA, M.C. Estimativa da área foliar e capacidade de retenção de calda fitossanitária em cafeeiro. Bioscience Journal, Uberlândia, v.24, p.66-73, 2008.
VALE, F.X.R.; FERNANDES FILHO, E.I.; LIBERATO, J.R.; ZAMBOLIM, L. Quant: A software to quantify plant disease severity. In: INTERNATIONAL WORKSHOP ON PLANT DISEASE EPIDEMIOLOGY, 2001, Ouro Preto. Proceedings... Ouro Preto: The International Society of Plant Pathology, 2008. v.8, p.160.

VARGAS, L.; ROMAN, E.S. Conceitos e aplicações dos adjuvantes. Passo Fundo: Embrapa Trigo, 2006. 10p. Disponível em: <http://www.cnpt.embrapa.br/ biblio/do/p_do56.htm>. Acesso em: 19 nov. 2008. 